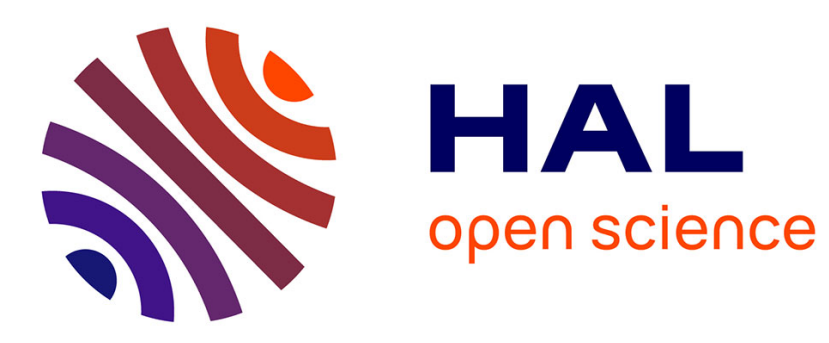

\title{
Blind estimation of the time-frequency activity rate of OFDMA signals
}

Mohamed Rabie Oularbi, François-Xavier Socheleau, Abdeldjalil Aissa El Bey, Sébastien Houcke

\section{- To cite this version:}

Mohamed Rabie Oularbi, François-Xavier Socheleau, Abdeldjalil Aissa El Bey, Sébastien Houcke. Blind estimation of the time-frequency activity rate of OFDMA signals. ICUMT 2010: International Conference on Ultra Modern Telecommunications, Oct 2010, Moscou, Russia. hal-00565928

\section{HAL Id: hal-00565928 \\ https://hal.science/hal-00565928}

Submitted on 15 Feb 2011

HAL is a multi-disciplinary open access archive for the deposit and dissemination of scientific research documents, whether they are published or not. The documents may come from teaching and research institutions in France or abroad, or from public or private research centers.
L'archive ouverte pluridisciplinaire HAL, est destinée au dépôt et à la diffusion de documents scientifiques de niveau recherche, publiés ou non, émanant des établissements d'enseignement et de recherche français ou étrangers, des laboratoires publics ou privés. 


\title{
Blind Estimation of The Time-Frequency Activity Rate of OFDMA Signals
}

\author{
Mohamed Rabie Oularbi, Francois-Xavier Socheleau, Abdeldjalil Aïssa-El-Bey and Sebastien Houcke \\ Institut TELECOM, Telecom Bretagne, UMR CNRS 3192 Lab-STICC \\ Université Europenne de Bretagne, France \\ Email : \{firstname.familyname\}@telecom-bretagne.eu
}

\begin{abstract}
The emerging trend to provide users with ubiquitous seamless wireless access leads to the development of multi-mode terminals able to smartly switch between heterogeneous wireless networks. This switching process known as vertical handover requires the terminal to first measure various network metrics. A relevant metric is the allocation rate of network physical channels. In the context of OFDMA and based on the physical signal properties, we propose a method to estimate this rate. Detailed numerical examples demonstrate the efficiency of the proposed estimator. Comparisons with a classical energy detector are also returned.
\end{abstract}

Index Terms-Vertical handover, time-frequency activity rate, OFDMA.

\section{INTRODUCTION}

In parallel to the deployment of new mobile services, there is an emerging trend to provide users with ubiquitous wireless access. This can be made possible by taking advantage of the coexistence of complementary heterogeneous networks such as 3G(LTE), WiMAX, Wifi etc. In such environment, a challenge is to develop multi-mode terminals able to smartly switch from one wireless interface to another while maintaining IP or voice connectivity and required QoS. This switching process is known as vertical handover.

Unlike the horizontal handover between two base stations using the same technology, the signal strength metrics are not suited to trigger a vertical handover. In such a case, metrics based on the downlink effective bandwidth or throughput are preferred. As detailed in [1], this effective bandwidth can be estimated from the data and the packet error rate that can be deduced from the Signal-to-Interference plus Noise Ratio and from the allocation rate of the cell's physical channels. Orthogonal Frequency-Division Multiple Access (OFDMA) is a multi-user version of the popular Orthogonal frequencydivision multiplexing (OFDM) digital modulation scheme. Multiple access is achieved in OFDMA by assigning subsets of subcarriers to individual users in a given time-slot, in view to support differentiated Quality of Service (QoS), i.e. to control the data rate and error probability individually for each user. When the number of active subcarriers is small, the data traffic should also be. Therefore, providing a satisfying downlink signal strength, it is better for a multimode-terminal to connect on such a base station rather than on one where the data traffic is high (high number of active subcarrier).
In this paper, we focus on the passive estimation of the allocation rate of OFDMA physical channels time-frequency slots. The allocation rate is defined as the number of active slots (allocated symbols) divided by the total number of slots per frame. In the context of vertical handover, only the passive estimation is relevant since the terminal seeks to know a priori if a network satisfies its bandwidth needs and not after wasting time and power to get connected to this network.

In some networks such as WiMAX, the physical channels allocation rate is regularly broadcasted by the base station so that it can be known by any terminal. However, this requires a multi-mode terminal that listens to the surrounding networks to intercept every frame preamble. If the multi-mode terminal has to decode every intercepted preamble to get this information, the vertical handover can be a very time and power consuming process.

An alternative approach developed in this contribution is to get the OFDMA physical channels allocation rate by blindly estimating the time-frequency activity rate of OFDMA physical signals. Such approach focuses on the signal properties and therefore does not require any message decoding (assuming this message is made available by the base station which may not be the case in all OFDMA networks). To the best of our knowledge, there is no algorithm published to date that addresses the blind estimation of the time-frequency activity rate of OFDMA signals.

The paper is organized as follows: Section II presents the OFDMA signal model. The proposed estimation algorithm is detailed in section III. The method is based on the estimation of the first and second order moments of the received signal. The performance of this estimator is assessed through simulations in Section IV. Finally, conclusions are presented in Section V.

\section{SIGNAL MODEL}

Assuming that an OFDMA symbol consists of up to $N$ active subcarriers, the discrete-time baseband equivalent transmitted signal is given by

$$
\begin{gathered}
x(m)=\sqrt{\frac{E_{s}}{N}} \sum_{k \in \mathbb{Z}} \sum_{n=0}^{N-1} \varepsilon_{k, n} a_{k, n} e^{2 i \pi \frac{n}{N}(m-D-k(N+D))} \\
. g(m-k(N+D)) .
\end{gathered}
$$


$E_{s}$ is the signal power, $a_{k, n}$ are the transmitted data symbols at the $n$-th subcarrier of the $k$-th ODFM block. These data symbols are assumed to be independent identically distributed (i.i.d), $D$ is the cyclic prefix length; $m \mapsto g(m)$ is the pulse shaping filter. $\varepsilon_{k, n}$ is a set of i.i.d random variable valued in $\{0,1\}$, expressing the absence or presence of signal activity in the $(k, n)$ time frequency slot.

Let $h(l)_{l=0, \ldots, L-1}$ be the baseband equivalent discrete-time channel impulse response of length $L$, such that $L+1<D$. The received samples of the OFDMA signals are then expressed as

$$
y(m)=\sum_{l=0}^{L} h(l) x(m-l)+w(m)
$$

where $w(m)$ is a zero mean circularly-symmetric complexvalued white Gaussian noise of variance $\sigma_{w}^{2}$. We assume that the receiver is synchronized with the transmitter in time and in frequency. This synchronization can be realized thanks to the frame preamble or thanks to blind techniques presented in [2] and [3]. We also assume that the noise variance is known or at least estimated thanks to blind method such as those detailed in [4], [5].

\section{Estimation Algorithm}

The estimation of the time-frequency activity rate $\alpha$ is equivalent to detect the active slots from the non active ones.

$$
\alpha=\frac{\sum_{k, n} I\left(\varepsilon_{k, n}=1\right)}{M N}
$$

where $I(A)$ is the indicator function of any event $A$ and $M$ is the number of observed OFDM symbols. Intuitively, considering that $\sigma_{w}^{2}$ is known, a classic detector structure could be used so that

$$
\hat{\alpha}=\frac{\sum_{k, n} I\left(\left|Y_{k, n}\right|>s\left(\sigma_{w}\right)\right)}{M N}
$$

where $s\left(\sigma_{w}\right)$ is a threshold function and $Y_{k, n}$ is the signal observation on the slot $(k, n)$.

$$
\begin{aligned}
Y_{k, n} & \triangleq \frac{1}{\sqrt{N}} \sum_{m=0}^{N-1} y[k(N+D)+D+m] e^{-2 i \pi \frac{n m}{N}} \\
& =\varepsilon_{k, n} a_{k, n} H_{k, n} \sqrt{E_{s}}+W_{k, n}
\end{aligned}
$$

where $H_{k, n}$ and $W_{k, n}$ are respectively the channel frequency response at subcarrier $n$ and the noise at subcarrier $n$ of the $k$-th received symbol.

The limitation of such approach is that the performance are strongly impacted by the choice of a threshold. In order to avoid this constraint, we hereafter propose an alternative method.

The proposed technique relies on the first and second order moments estimate of the observed samples absolute value. This moments are indeed dependent of the activity rate $\alpha$.

For all $(k, n)$ such that $\varepsilon_{k, n}=0$, the observations are made of noise-only slots such that they satisfy $Y_{k, n} \sim \mathcal{C N}\left(0, \sigma_{w}^{2}\right)$.
Therefore, in this case the absolute value $\left|Y_{k, n}\right|$ has a Rayleigh distribution of expectation

$$
\mathbb{E}\left[\left|Y_{k, n}\right| / \varepsilon_{k, n}=0\right]=\frac{\sqrt{\pi}}{2} \sigma_{w}
$$

where $\mathbb{E}[. /$.$] defines the conditional expectation.$

When the observations are made of signal plus noise samples (i.e $\varepsilon_{k, n}=1$ ), their distribution is quite difficult to know in a context of passive estimation. The various $a_{k, n}$ can indeed have different distributions that depend on the symbols constellation (usually BPSK, QPSK, 16-QAM or 64QAM [6]). Actual systems employing adaptive modulation, this constellation can be different from a slot to another. Therefore, according to the principle of maximum entropy [7], the state of ignorance on the constellation distribution is here modeled by a uniform law. Hence, without prior information, we assume that the probability to get each constellation equals $1 / 4$ (note that the impact of this assumption is discussed in section 4). Consequently the expectation of $\left|Y_{k, n}\right|$ when $\varepsilon_{k, n}=1$ can be written as

$$
\begin{aligned}
\mathbb{E}\left[\left|Y_{k, n}\right|\right. & \left./ \quad \varepsilon_{k, n}=1\right]=\mathbb{E}\left[\left|a_{k, n} H_{k, n} \sqrt{E_{s}}+W_{k, n}\right|\right] \\
& =\frac{1}{4} \sum_{j=1}^{4} \mathbb{E}\left[\left|a_{k, n} H_{k, n} \sqrt{E_{s}}+W_{k, n}\right| / a_{k, n} \in \mathcal{C}_{M_{j}}\right]
\end{aligned}
$$

where the $C_{M_{j}}$ constellations are $M_{j}$-QAM such that for $j=$ $1, \ldots, 4 M_{j}$ is respectively equal to $2,4,16,64$.

Assuming a Gaussian noise, a Rayleigh fading channel and a known $a_{k, n}$, the distribution of the observed slots is Gaussian: $Y_{k, n} / a_{k, n}, \varepsilon_{k, n}=1 \sim \mathcal{C N}\left(0, \sum_{0}^{L-1} \sigma_{h(l)}^{2} E_{s}\left|a_{k, n}\right|^{2}+\sigma_{w}^{2}\right)$. It then follows that the absolute value $\left|Y_{k, n} / a_{k, n}, \varepsilon_{k, n}=1\right|$ has a Rayleigh distribution. After performing integration over all the possible values of $a_{k, n}$ in each $C_{M_{j}}$ constellation noted $c_{p}$ we find that

$$
\begin{aligned}
& \mathbb{E}\left[\left|Y_{k, n}\right| / \varepsilon_{k, n}=1\right]= \\
& \quad \frac{\sqrt{\pi}}{2} \frac{1}{4} \sum_{j=1}^{4} \frac{1}{M_{j}} \sum_{p=1}^{M_{j}} \sqrt{\sum_{l} \sigma_{h(l)}^{2} E_{s}\left|c_{p}\right|^{2}+\sigma_{w}^{2}}
\end{aligned}
$$


and consequently,

$$
\begin{aligned}
\mathbb{E}[ & \left.Y_{k, n} \mid / \varepsilon_{k, n}=1\right]= \\
\frac{\sqrt{\pi}}{8}[ & \frac{5}{2} \sqrt{\sum_{l} \sigma_{h(l)}^{2} E_{s}+\sigma_{w}^{2}}+\frac{1}{4} \sqrt{\sum_{l} \sigma_{h(l)}^{2} \frac{E_{s}}{5}+\sigma_{w}^{2}} \\
& +\frac{1}{4} \sqrt{\frac{9}{5} \sum_{l} \sigma_{h(l)}^{2} E_{s}+\sigma_{w}^{2}}+\frac{1}{16} \sqrt{\sum_{l} \sigma_{h(l)}^{2} \frac{E_{s}}{21}+\sigma_{w}^{2}} \\
& +\frac{1}{8} \sqrt{\frac{5}{21} \sum_{l} \sigma_{h(l)}^{2} E_{s}+\sigma_{w}^{2}}+\frac{1}{16} \sqrt{\frac{3}{7} \sum_{l} \sigma_{h(l)}^{2} E_{s}+\sigma_{w}^{2}} \\
& +\frac{1}{8} \sqrt{\frac{13}{21} \sum_{l} \sigma_{h(l)}^{2} E_{s}+\sigma_{w}^{2}}+\frac{1}{8} \sqrt{\frac{17}{21} \sum_{l} \sigma_{h(l)}^{2} E_{s}+\sigma_{w}^{2}} \\
& +\frac{3}{16} \sqrt{\frac{25}{21} \sum_{l} \sigma_{h(l)}^{2} E_{s}+\sigma_{w}^{2}}+\frac{1}{8} \sqrt{\frac{29}{21} \sum_{l} \sigma_{h(l)}^{2} E_{s}+\sigma_{w}^{2}} \\
& +\frac{13}{8} \sqrt{\frac{37}{21} \sum_{l} \sigma_{h(l)}^{2} E_{s}+\sigma_{w}^{2}}+\frac{13}{16} \sqrt{\frac{7}{3} \sum_{l} \sigma_{h(l)}^{2} E_{s}+\sigma_{w}^{2}} \\
& =\varphi\left(\sum_{l} \sigma_{h(l)}^{2} E_{s}\right)
\end{aligned}
$$

where $\varphi$ is a function that associate to each $E_{s}$ the expectation $\mathbb{E}\left[\left|Y_{k, n}\right| / \varepsilon_{k, n}=1\right]$, when $\sigma_{w}^{2}$ is assumed to be known.

Since $\alpha \%$ of the slots are active and $(1-\alpha) \%$ are not, the expectation of the overall observed samples module is expressed as

$$
\mathbb{E}\left[\left|Y_{k, n}\right|\right]=\alpha \varphi\left(\sum_{l} \sigma_{h(l)}^{2} E_{s}\right)+(1-\alpha) \frac{\sqrt{\pi}}{2} \sigma_{w}
$$

Moreover, the second order moment $\mathbb{E}\left[\left|Y_{k, n}\right|^{2}\right]$ satisfies

$$
\mathbb{E}\left[\left|Y_{k, n}\right|^{2}\right]=\sigma_{w}^{2}+\alpha \sum_{l} \sigma_{h(l)}^{2} E_{s}, \forall \varepsilon_{k, n}
$$

It follows that $\sum_{l} \sigma_{h(l)}^{2} E_{s}$ is deduced as

$$
\sum_{l} \sigma_{h(l)}^{2} E_{s}=\frac{\mathbb{E}\left[\left|Y_{k, n}\right|^{2}\right]-\sigma_{w}^{2}}{\alpha},
$$

If we denote by $m_{1}=\mathbb{E}\left[\left|Y_{k, n}\right|\right]$ and $m_{2}=\mathbb{E}\left[\left|Y_{k, n}\right|^{2}\right]$ then

$$
\begin{gathered}
\hat{m}_{1}=\frac{1}{M N} \sum_{k=0}^{M-1} \sum_{n=0}^{N-1}\left|Y_{k, n}\right| \\
\hat{m}_{2}=\frac{1}{M N-1} \sum_{k=0}^{M-1} \sum_{n=0}^{N-1}\left|Y_{k, n}\right|^{2}
\end{gathered}
$$

substituting this value in equation (10), an estimate of the channel occupancy rate $\hat{\alpha}$ can be obtained solving the following equation

$$
\hat{\alpha} \varphi\left(\frac{\hat{m}_{2}-\sigma_{w}^{2}}{\hat{\alpha}}\right)+(1-\hat{\alpha}) \frac{\sqrt{\pi}}{2} \sigma_{w}-\hat{m}_{1}=0
$$

This equation has no analytical solution, we propose to solve it by a binary search algorithm. The whole corresponding technique is presented in algorithm 1 . Notice that no channel estimation is required.

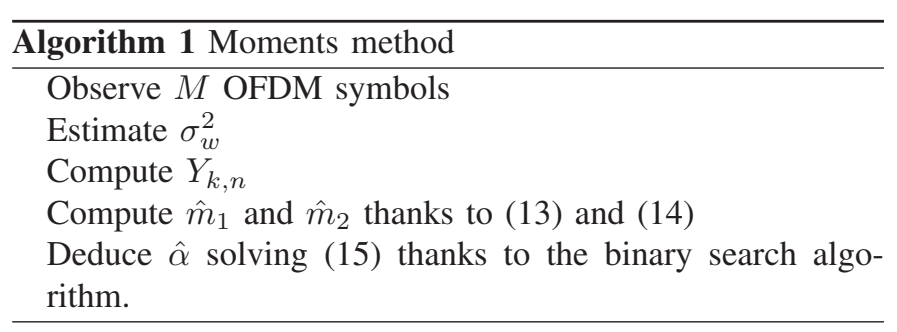

\section{Simulations}

In this section, OFDMA signals with 512 subcarriers are considered. $D$ is set to $128, M=24$ and the time-frequency slots allocation is supposed i.i.d. Each $a_{k, n}$ is randomly chosen within BPSK, QPSK, 16-QAM and 64-QAM constellations according to a uniform law. These constellations are the main constellations used by the WiMAX Adaptive Modulation and Coding (AMC) [6].

The estimator performance is assessed in WMAN environment where the channel is highly selective [8]. Figure 1 shows the NMSE (Normalized Mean Square Error) of the proposed estimators for different SNR versus the activity rate. The SNR is defined as

$$
\mathrm{SNR}=\frac{S}{\sigma_{w}^{2}}
$$

with

$$
S=E_{s} \mathbb{E}\left[\left|\varepsilon_{k, n} a_{k, n}\right|^{2}\right] \sum_{l=0}^{L-1} \sigma_{h(l)}^{2}
$$

The propagation channel $\left\{h_{k}(l)\right\}_{l=0, \cdots, L}$ has an exponential decay profile for its non-null component (i.e., $\mathbb{E}\left[\left|h_{k}(l)\right|^{2}\right]=$ $G e^{-l / \mu}$ for $\left.l=0, \cdots, L\right)$ with $L=D, \mu=32$ and $G$ is chosen such that $\sum_{l=0}^{L} \mathbb{E}\left[\left|h_{k}(l)\right|^{2}\right]=1$. The channel is assumed to be time variant with a Doppler frequency equal to $100 \mathrm{~Hz}$. Figure 1 compares the performance of the estimator in the two cases where $\sigma_{w}$ is first assumed to be perfectly known and when it is estimated thanks to the method presented in [5] and [4]. We can observe, that the estimator's performance deteriorates when $\sigma_{w}$ is estimated, but still offers satisfying performance for the targeted application. A NMSE of $-15 \mathrm{~dB}$ can indeed be considered as sufficiently accurate to decide whether to trigger a handover or not.

In figure 2, the performance of the proposed estimator is compared to the Constant false alarm rate (CFAR) [9] energy detector per slot. This classical approach, relies on a threshold associated to a false alarm $P_{f a}$. It assumes that $\varepsilon_{k, n}=1$ if

$$
\left|Y_{k, n}\right|^{2}>-\sigma_{w}^{2} \ln \left(P_{f a}\right)
$$

The results show the problems induced by the choice of the threshold. We can clearly observe that for a given $\mathrm{SNR}=10 \mathrm{~dB}$, the choice of the threshold greatly impacts the 


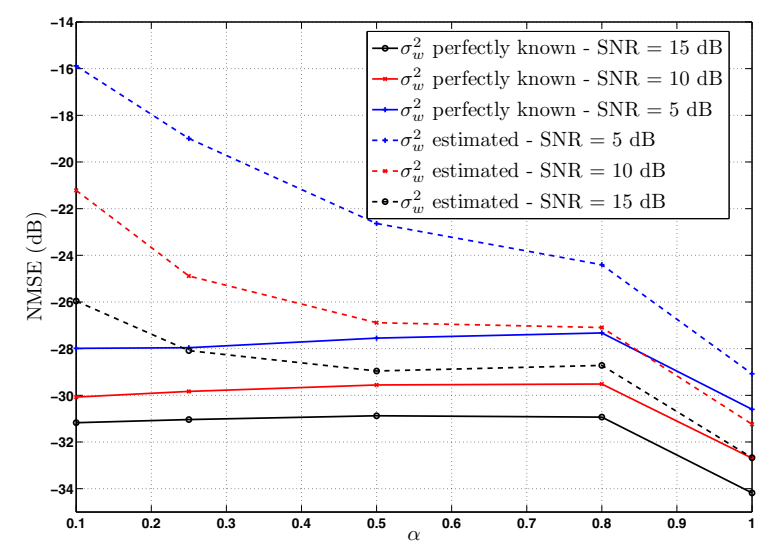

Fig. 1. NMSE of the proposed method versus the activity rate, $\sigma_{w}$ known and estimated.

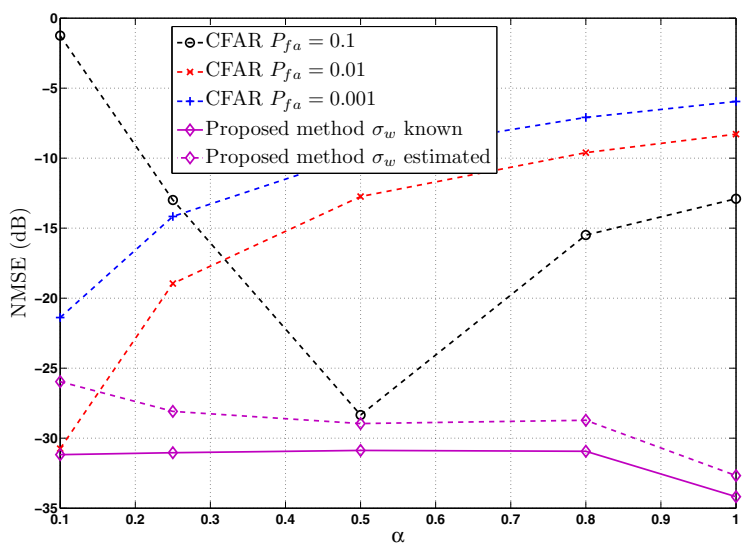

Fig. 2. NMSE of the proposed method compared to the CFAR method

performance of the CFAR method. The proposed approach offers better and more stable results even when $\sigma_{w}$ is estimated.

Figure 3, compares the performances of the proposed algorithm in the case where the $a_{k, n}$ are not uniformly chosen from one of the possible constellation present in WiMAX. Indeed, we assess the performance when the probability that a chosen symbol belongs to any constellation is $\mathrm{P}\left(a_{k, n} \in \mathrm{BPSK}\right)=$ $\frac{1}{10}, \mathrm{P}\left(a_{k, n} \in \mathrm{QPSK}\right)=\frac{1}{10}, \mathrm{P}\left(a_{k, n} \in 16-\mathrm{QAM}\right)=\frac{4}{10}$, $\mathrm{P}\left(a_{k, n} \in\right.$ 64-QAM $)=\frac{4}{10}$ whereas the receiver expect a uniform distribution. We can clearly note that the proposed algorithm is robust to a non-equirepartition of the constellation due to the AMC scheme.

\section{CONCLUSiON}

In this paper, a new blind estimation method of OFDMA time-frequency activity rate has been presented. The method is computationally cheap and exhibits good performance for vertical handover. This approach does not rely on a choice of a threshold and show good results compared to the classical CFAR energy detector even when $\sigma_{w}$ is estimated.

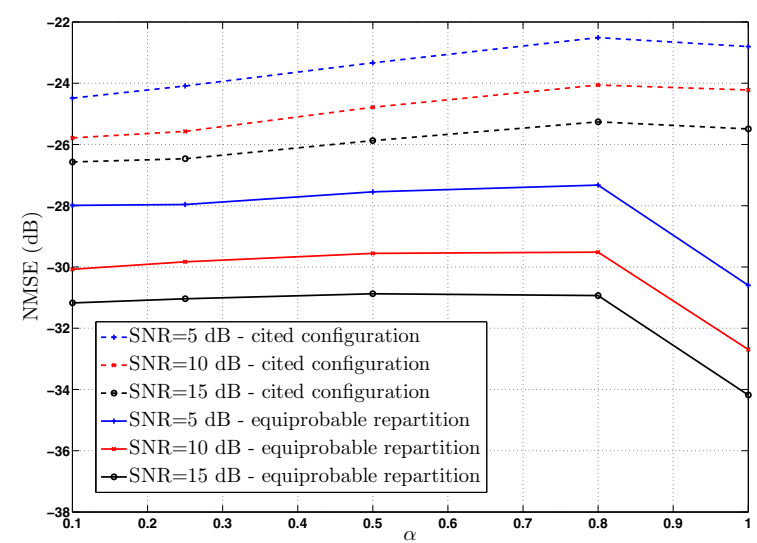

Fig. 3. NMSE of the proposed method in the case of uniform and nonuniform repartition of the constellations.

\section{REFERENCES}

[1] Z. Dai, R. Fracchia, J. Gosteau, P. Pellati, and G. Vivier, "Vertical handover criteria and algorithm in IEEE 802.11 and 820.16 hybrid networks," ICC, pp. 2480-2484, 2008.

[2] J. van de Beek, M.Sandell, and P.Borjesson, "ML estimation of time and frequency offset in OFDM systems," IEEE Transactions on Acoustics, Speech, and Signal Processing,, vol. 45, pp. 1800-1805, 1997.

[3] B.Park, E.Ko, H.Cheon, C.Kang, and D.Hong, "A blind OFDM synchronization algorithm based on cyclic correlation," IEEE Globecom conf, vol. 5, pp. 3116-3119, 2001.

[4] F.-X.Socheleau, D. Pastor, A. Aissa-El-Bey, and S.Houcke, "Blind noise variance estimation for OFDMA signals," ICASSP, pp. 2581 - 2584, 2009.

[5] F.-X. Socheleau, A. Aissa-El-Bey, and S. Houcke, "Non Data-Aided SNR Estimation of OFDM Signals," IEEE communications letters, vol. 12, no. $11,2008$.

[6] IEEE Std 802.16, "Part 16: air interface for broadband wireless access systems, Amendment 2 : Physical and Medium Access Control layers for Combined Fixed and Mobile Operation in License Bands and Corrigendum 1," 2005.

[7] E. T. Jaynes, Probability Theory: The Logic of Science., Addison Wesley, NY, 2000.

[8] V. Erceg et al, Channel Models for Fixed Wireless Applications, IEEE 802.16 Broadband Wireless Access Working Group, 2001.

[9] L. Scharf, Statistical Signal Processing: Detection, Estimation, and Time Series Analysis., Addison Wesley, NY. 